\title{
Retrospective Diagnosis of COVID-19 in an Asymptomatic Patient Undergoing Emergency Surgery
}

\author{
Margaret Yanfong Chong, Daphne Xin Ying Moo \\ Department of Anaesthesiology and Intensive Care, Changi General Hospital, Singapore \\ Email: margaretyfc@gmail.com
}

How to cite this paper: Chong, M.Y. and Moo, D.X.Y. (2020) Retrospective Diagnosis of COVID-19 in an Asymptomatic Patient Undergoing Emergency Surgery. Open Journal of Anesthesiology, 10, 277-283. https://doi.org/10.4236/ojanes.2020.108024

Received: July 20, 2020

Accepted: August 17, 2020

Published: August 20, 2020

Copyright (c) 2020 by author(s) and Scientific Research Publishing Inc. This work is licensed under the Creative Commons Attribution International License (CC BY 4.0).

http://creativecommons.org/licenses/by/4.0/

\begin{abstract}
Background: With reports of higher mortality and complications occurring in patients with perioperative 2019 novel coronarvirus disease (COVID-19), most elective surgeries have been postponed. However, evidence regarding emergency surgeries in patients with COVID-19 remains scarce. We report the case of a patient with asymptomatic perioperative COVID-19, presenting with an acute abdomen requiring surgery. Case: A 25 -year-old male, with a prior nasopharyngeal swab that was negative for SARS-CoV-2, presented with classical signs and symptoms of acute appendicitis. Clinical examination and investigations were not suggestive of COVID-19 infection. He underwent laparoscopic appendicectomy with infection control precautions. Postoperatively, he was found to be positive for SARS-CoV-2 but remained asymptomatic and had an uneventful recovery. Conclusion: In asymptomatic individuals with higher risks, negative test results should be viewed cautiously. The benefits of urgent surgical interventions must be weighed against the risks of complications due to perioperative COVID-19 in these patients.
\end{abstract}

\section{Keywords}

Coronavirus, COVID-19, Asymptomatic, Emergency Surgery, Infection Control, Pharyngeal Swab, Personal Protective Equipment

\section{Introduction}

The 2019 novel coronavirus disease (COVID-19) has become a global health crisis since its initial outbreak. Besides the typical manifestations of fever, respiratory symptoms, myalgia, lymphopaenia and radiological evidence of pneumonia, asymptomatic infections have also been reported [1]. With presumed asymptomatic transmission of COVID-19 during the incubation period [2], prevention 
of widespread transmission is challenging. In view of the massive burden on medical systems worldwide caused by the outbreak [3], as well as reports of postoperative pulmonary complications and higher mortality in patients with perioperative COVID-19 [4], most elective surgeries have been postponed. However, evidence regarding patients with COVID-19 and concomitant conditions requiring urgent surgical interventions remains scarce. The principle concern is to balance the benefits of timely surgical management against the risks of complications resulting from perioperative COVID-19 [5].

We report the case of an individual considered high risk for COVID-19, but had a previous negative swab and no respiratory symptoms, who developed acute suppurative appendicitis and was subsequently found to be positive for the coronavirus SARS-CoV-2 after appendicectomy.

\section{Case Report}

A previously well 25-year-old Bangladeshi male staying in a foreign worker dormitory (FWD) in Singapore, presented with a three-day history of fever, abdominal pain, vomiting and diarrhoea. His abdominal pain started in the epigastrium and migrated to the right iliac fossa (RIF). No symptoms of an acute respiratory illness (ARI) were present. Table 1 summarises his sociodemographic and clinical characteristics. His FWD was not labelled as a COVID-19 cluster by the Ministry of Health $(\mathrm{MOH})$ and he had a negative SARS-CoV-2 polymerase chain reaction (PCR) nasopharyngeal swab 14 days ago. Since then, he only ventured out of his dormitory room to collect food or use the common lavatory.

The patient presented with a fever of $38.1^{\circ} \mathrm{C}$ and a pulse rate of 112 beats per minute. Blood pressure was $121 / 76 \mathrm{mmHg}$ and he had an oxygen saturation of $98 \%$ on room air. Significant physical findings included clinical signs of dehydration and RIF tenderness with generalised abdominal guarding. Examination of the lungs revealed equal bilateral air entry and no adventitious lung sounds. Laboratory investigations showed haemoglobin level of $16.5 \mathrm{~g} / \mathrm{dL}$ and leukocyte count of $15.1 \times 10^{3} / \mathrm{uL}$ with neutrophilia (86.2\%). Absolute lymphocyte count was normal at $1.2 \times 10^{3} / \mathrm{uL}$. There was evidence of acute kidney injury (AKI), with an estimated glomerular filtration rate of $56 \mathrm{ml} / \mathrm{min} / 1.72 \mathrm{~m}^{2}$ and serum creatinine was $148 \mathrm{umol} / \mathrm{L}$. His chest X-ray did not show any abnormality.

Table 1. Socioeconomic and clinical characteristics of patient.

\begin{tabular}{cc}
\hline Age & 25 years \\
Gender & Male \\
Nationality & Bangladeshi \\
Occupation & Foreign worker \\
Residence & Foreign worker dormitory \\
Past medical history & None \\
Symptoms & Fever, epigastric pain migrating to right iliac fossa, vomiting, diarrhoea \\
\hline & \\
\hline
\end{tabular}


In view of his place of residence in a FWD, he was deemed as high risk for COVID-19, despite a prior negative swab [6]. A combined nasopharyngeal and oropharyngeal swab was taken and patient was placed into a ward designated for all patients with ARI.

A computed tomography scan of the abdomen and pelvis (CTAP) was initially ordered to investigate his abdominal pain. Subsequently, the surgeon decided that in light of the classical signs and symptoms present, a clinical diagnosis would suffice. Further radiological evidence and the need for confirmatory COVID-19 swab results before proceeding with an appendicectomy would delay surgery with no additional benefit to the patient. Since COVID-19 infection could not be completely ruled out, the surgical and anaesthetic teams agreed that the surgery would be performed in the designated operating room (OR) away from the main surgical theatres. The number of healthcare workers (HCW) involved was limited to the minimum required, and full personal protective equipment (PPE) was mandatory.

Prior to the transfer of the patient, signage was put up in the surgical theatre complex to divert human traffic. All required equipment and drugs were prepared beforehand. To achieve the highest chance of a single successful attempt at intubation, the McGrath ${ }^{\text {Tx }}$ Video-laryngoscope with a disposable blade was used. After adequate pre-oxygenation, rapid sequence induction with midazolam 1.5 $\mathrm{mg}$, propofol $170 \mathrm{mg}$, fentanyl $100 \mathrm{mcg}$ and suxamethonium $100 \mathrm{mg}$ was performed. Intra-operatively, the surgeon found a suppurative non-perforated appendix with pus. The surgery lasted 35 minutes and was uneventful. He was maintained on desflurane and given $8 \mathrm{mg}$ morphine, paracetamol and local anaesthetic infiltration for analgesia. All HCW involved kept full PPE on throughout surgery.

Postoperatively, he was extubated only in the presence of the anaesthetic team. After extubation, he was monitored in the OR for 30 minutes and was transferred directly back to his assigned ward wearing a surgical face mask. His pharyngeal swab subsequently detected SARS-CoV-2 and he was transferred to the designated COVID-19 ward. His renal function normalised on post-operative day (POD) 1. His vital signs remained stable and he remained afebrile throughout the admission. Table 2 shows the comparison of laboratory results immediately before surgery and POD 1 . On POD 2, he remained asymptomatic and was transferred to a community isolation facility. He returned for his follow-up with the surgical team 6 weeks later, without any postoperative surgical or pulmonary complication.

\section{Discussion}

In this retrospectively diagnosed COVID-19 patient who underwent emergency surgery unrelated to his COVID-19 infection, there are several lessons we learned.

Firstly, in a patient with fever from an apparent alternative source, COVID-19 should still be considered in the setting of this pandemic. This patient presented 
Table 2. Pre- and post-operative laboratory results.

\begin{tabular}{ccc}
\hline & Pre-operative $(19 / 5 / 20)$ & POD1 $(20 / 5 / 20)$ \\
\hline $\mathrm{U}(\mathrm{mmol} / \mathrm{L})$ & 5.3 & 4.5 \\
$\mathrm{Na}(\mathrm{mmol} / \mathrm{L})$ & 134 & 3.6 \\
$\mathrm{~K}(\mathrm{mmol} / \mathrm{L})$ & 3.4 & 98 \\
$\mathrm{Cl}(\mathrm{mmol} / \mathrm{L})$ & 94 & 26 \\
$\mathrm{Bicarb}(\mathrm{mmol} / \mathrm{L})$ & 24 & 5.4 \\
$\mathrm{Glucose}(\mathrm{mmol} / \mathrm{L})$ & 7.4 & 84 \\
$\mathrm{Cr}(\mathrm{umol} / \mathrm{L})$ & 148 & $>90$ \\
$\mathrm{eGFR}\left(\mathrm{ml} / \mathrm{min} / 1.73 \mathrm{~m}^{2}\right)$ & 56 & \\
$\mathrm{CRP}$ & 48.4 & \\
$\mathrm{Hb}(\mathrm{g} / \mathrm{dL})$ & 16.5 & \\
$\mathrm{WBC}\left(\times 10^{3} / \mathrm{uL}\right)$ & 15.1 & \\
$\mathrm{Plt}\left(\times 10^{3} / \mathrm{uL}\right)$ & 164 & \\
$\mathrm{ALC}\left(\times 10^{3} / \mathrm{uL}\right)$ & 1.2 & \\
\hline
\end{tabular}

with the classical picture of acute appendicitis, with no symptoms of ARI or lymphopaenia, and a normal chest X-ray. In view of his worsening sepsis, the surgical team felt that emergency surgery without confirmatory radiological evidence was warranted. It remains hypothetical whether ground glass opacities and/or consolidation could have been detected on a pre-operative CTAP. A case series described three patients without respiratory symptoms who underwent CTAP for abdominal pain, and CT evaluation of the lung bases showed groundglass opacities. These patients were subsequently diagnosed with COVID-19 [7]. However, a recent systematic review and meta-analysis found that CT findings lack specificity in differentiating different types of pneumonia, and normal CT findings were reported in $47.3 \%$ of studies [8]. As the pandemic shows no sign of abating, a high index of suspicion must remain for all febrile patients who have been stratified as moderate to high risk for COVID-19, even in the presence of another fever source.

Secondly, this patient had a negative swab for SARS-CoV-2 prior to his current presentation. This was done as part of the proactive screening programme conducted by $\mathrm{MOH}$, in an attempt to identify and isolate infected individuals early, as well as determine the extent of COVID-19 infection in FWD. It would be difficult to determine if the previous surveillance swab was a false negative result or if the patient only contracted the disease subsequently in the dormitory. False-negative results can be contributed by multiple causes. The sensitivity of reverse transcription-PCR depends on several factors including sampling technique, timing and site of sampling, viral load and mutations in the viral genome [9] [10]. It could also be due to the inherent nature of coronavirus itself [11] [12]. As these diagnostic tests become more readily available, understanding the 
principles behind them becomes as important as the result itself. A false-negative result provides deceptive reassurance, and this has implications on both personal and public health. For individuals considered to be in higher risk groups for COVID-19, negative test results should be interpreted cautiously [13]. If COVID19 infection cannot be ruled out completely, the highest level of protection should be adopted [14]. In this case, none of the HCW were infected despite the absence of powered air-purifying respirators (PAPR).

Thirdly, the decision to proceed with surgery in a case with high suspicion of COVID-19 should be made jointly by the anaesthetic and surgical teams. A mortality rate of $20.5 \%$ has been reported in a retrospective cohort study involving 34 patients with perioperative COVID-19, with $44.1 \%$ of them requiring intensive care [15]. Their data suggested that surgery may accelerate and exacerbate disease progression of COVID-19, likely due to surgical stress causing immune function impairment and early systemic inflammatory response. An international cohort study [4] also reported that post-operative complications occurred in half of the patients with perioperative SARS-CoV-2 infection and these were associated with high mortality. It concluded that non-urgent procedures should be postponed, and non-operative treatment should be considered during this pandemic. Mortality was particularly high for men aged 70 years or older who had emergency or major elective surgeries. However, there was no mortality reported for patients less than 29 years old in that study. This is consistent with our young patient with no comorbidities, where the benefits of early source control preventing further worsening of intra-abdominal sepsis likely outweighed the risks of possible poor outcomes. Currently, evidence regarding outcomes of COVID-19 patients who required emergency surgeries remains limited.

Lastly, this case further reiterates the importance of infection control measures in the management of suspected or confirmed patients with COVID-19 coming for surgery, with the aim to minimize the transmission to HCW during aerosol-generating procedures in the OR. In this case, detailed protocols were present to ensure a swift and isolated patient transit. The number of HCW involved was minimised and all staff involved wore PPE (N95 masks, protective eyewear, gloves, disposable gowns and disposable shoe covers) during the perioperative management of the patient.

\section{Conclusion}

In conclusion, risk stratification is of utmost importance during a pandemic. In individuals with higher pre-test probability, COVID-19 should still be considered even in the presence of an alternative source of fever or the absence of respiratory symptoms. Stringent infection control measures remain paramount in the prevention of transmission to HCW.

\section{Conflicts of Interest}

The authors declare no conflicts of interest regarding the publication of this pa- 
per.

\section{References}

[1] Deng, L., Ji, L., Meng, Z., Gan, Y. and Cheng, G. (2020) Family Cluster of Asymptomatic Infections with COVID-19: A Case Series of 4 Patients. Quantitative Imaging in Medicine and Surgery, 10, 1127-1132.

https://doi.org/10.21037/qims.2020.04.15

[2] Bai, Y., Yao, L., Wei, T., Tian, F., Jin, D., Chen, L. and Wang, M. (2020) Presumed Asymptomatic Carrier Transmission of COVID-19. JAMA, 323, 1406. https://doi.org/10.1001/jama.2020.2565

[3] World Health Organisation COVID-19 Situation Reports. https://www.who.int/emergencies/diseases/novel-coronavirus-2019/situation-reports/

[4] Archer, J., Odeh, A., Ereidge, S., Salem, H., Jones, G., Gardner, A., Tripathi, S., Gregg, A., Jeganathan, R., Siddique, M., Lombardi, C., Martin, B., Leo, C., Dass, D., Di Franco, G., Jiao, L., Mari, G., Capitan-Morales, L., Connelly, T., Alanbuki, A., De Virgilio, A., Schilling, C., San Miguel Mendez, C., Kulkarni, G., Nizami, K., Walsh, S., Dean, H., Ruiz-Marin, M., Houston, R., Trompetto, M., Chrastek, D., Kouritas, V., Cannoletta, M., Rosato, F., Kaushal, M., Costa, P., Elkadi, H., Johnstone, J., Irvine, E., Alvarez, M., Corbellini, C., Venkatesan, G., Mateo-Sierra, O., MartínezPérez, C., Serrano González, J., Hernández Bartolomé, M., Díaz Pérez, D., Gutiérrez Samaniego, M., Galindo Jara, P. and Sharma, N. (2020) Mortality and Pulmonary Complications in Patients Undergoing Surgery with Perioperative SARS-CoV-2 Infection: An International Cohort Study. The Lancet, 396, 27-38.

[5] Besnier, E., Tuech, J. and Schwarz, L. (2020) We Asked the Experts: Covid-19 Outbreak: Is There Still a Place for Scheduled Surgery? Reflection from Pathophysiological Data. World Journal of Surgery, 44, 1695-1698.

https://doi.org/10.1007/s00268-020-05501-6

[6] Singapore Ministry of Health (2020) Circular 97A/2020 Revision of Suspect Case Definition for Coronavirus Disease 2019 (COVID-19).

[7] Siegel, A., Chang, P., Jarou, Z., Paushter, D., Harmath, C., Arevalo, J. and Dachman, A. (2020) Lung Base Findings of Coronavirus Disease (COVID-19) on Abdominal CT in Patients with Predominant Gastrointestinal Symptoms. American Journal of Roentgenology, 1-3. https://doi.org/10.2214/AJR.20.23232

[8] Sun, Z., Zhang, N., Li, Y. and Xu, X. (2020) A Systematic Review of Chest Imaging Findings in COVID-19. Quantitative Imaging in Medicine and Surgery, 10, 1058 1079. https://doi.org/10.21037/qims-20-564

[9] Ai, T., Yang, Z., Hou, H., Zhan, C., Chen, C., Lv, W., Tao, Q., Sun, Z. and Xia, L. (2020) Correlation of Chest CT and RT-PCR Testing in Coronavirus Disease 2019 (COVID-19) in China: A Report of 1014 Cases. Radiology, 296, E32-E40. https://doi.org/10.1148/radiol.2020200642

[10] Winichakoon, P., Chaiwarith, R., Liwsrisakun, C., Salee, P., Goonna, A., Limsukon, A. and Kaewpoowat, Q. (2020) Negative Nasopharyngeal and Oropharyngeal Swabs Do Not Rule Out COVID-19. Journal of Clinical Microbiology, 58, e0297-20. https://doi.org/10.1128/JCM.00297-20

[11] Peiris, J., Chu, C., Cheng, V., Chan, K., Hung, I., Poon, L., Law, K., Tang, B., Hon, T., Chan, C., Chan, K., Ng, J., Zheng, B., Ng, W., Lai, R., Guan, Y. and Yuen, K. (2003) Clinical Progression and Viral Load in a Community Outbreak of Coronavirus-Associated SARS Pneumonia: A Prospective Study. The Lancet, 361, 1767 1772. https://doi.org/10.1016/S0140-6736(03)13412-5 
[12] Memish, Z., Al-Tawfiq, J., Makhdoom, H., Assiri, A., Alhakeem, R., Albarrak, A., Alsubaie, S., Al-Rabeeah, A., Hajomar, W., Hussain, R., Kheyami, A., Almutairi, A., Azhar, E., Drosten, C., Watson, S., Kellam, P., Cotten, M. and Zumla, A. (2014) Respiratory Tract Samples, Viral Load, and Genome Fraction Yield in Patients with Middle East Respiratory Syndrome. Journal of Infectious Diseases, 210, 1590-1594. https://doi.org/10.1093/infdis/jiu292

[13] West, C., Montori, V. and Sampathkumar, P. (2020) COVID-19 Testing. Mayo Clinic Proceedings, 95, 1127-1129. https://doi.org/10.1016/j.mayocp.2020.04.004

[14] Gao, Y., Xi, H. and Chen, L. (2020) Emergency Surgery in Suspected COVID-19 Patients with Acute Abdomen. Annals of Surgery. https://doi.org/10.1097/SLA.0000000000003961

[15] Lei, S., Jiang, F., Su, W., Chen, C., Chen, J., Mei, W., Zhan, L., Jia, Y., Zhang, L., Liu, D., Xia, Z. and Xia, Z. (2020) Clinical Characteristics and Outcomes of Patients Undergoing Surgeries during the Incubation Period of COVID-19 Infection. EClinicalMedicine, 21, Article ID: 100331. https://doi.org/10.1016/j.eclinm.2020.100331 